\title{
Fingerprint Based Security System using GSM Module
}

\author{
Mr. Amit Hatekar ${ }^{1,}$ Harsh Babani ${ }^{2}$, Tejal Kakde ${ }^{2}$, Namit Wadhwa ${ }^{2}$ \\ ${ }^{1}$ Assistant Professor, Department of EXTC, Thadomal Shahani Engineering College, Mumbai, India. \\ ${ }^{2}$ Undergraduate Students, Department of EXTC, Thadomal Shahani Engineering College, Mumbai, India
}

\begin{abstract}
This paper mainly focuses on using wireless technology for security effectively. The system is SMS-based and uses biometric technology to revolutionize the standards of security. It uses a GSM Modem to send an SMS to the authorized person in case of an intrusion. The project is realized by interfacing a fingerprint sensor with a $89 \mathrm{c} 51$ microcontroller and a GSM Module. As the system uses GSM technology, it provides ubiquitous access to the system for security.

Index Terms: Bank Security, 89c51, GSM, GSM SIM 900.
\end{abstract}

\section{INTRODUCTION}

Now a day's safety has become an essential issue for most people. Increasing incidence of crimes against banks needed a serious re-look at the security arrangements and guidelines followed by the banks. This scenario demands compatible, efficient and reliable security and safety measures. Currently, Personal Identification Numbers, passwords or identification cards are used for personal identification. However, cards can be stolen; numbers and passwords can be guessed or forgotten.

We have tried to overcome these problems using application of biometrics which is fingerprint sensor, microcontroller, GSM Modem and a Relay circuit. When an unauthorized person access the fingerprint module, an interrupt is sent to the microcontroller which is further interfaced with a GSM module. It sends an SMS to the authorized person to alert him/her. The basic flow of this system is shown in Figure 1. Thus, this project takes some action after the intrusion has already taken place. This project gives the access of locker to only an authorized person and possible theft is immediately notified by using GSM module. This system is easy to install and can be used in any environment.

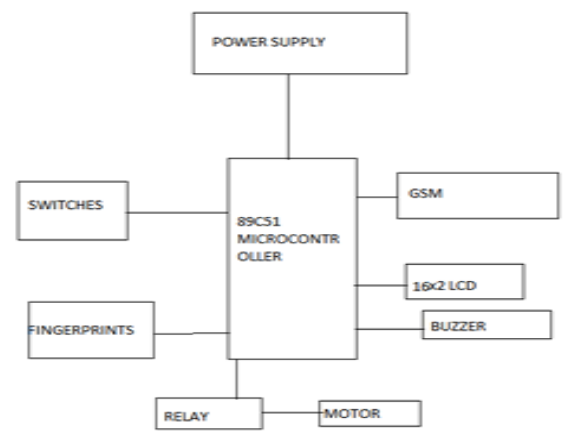

Figure 1: Block Diagram of Entire System

\section{FINGERPRINT MODULE}

Fingerprint sensor module R305 (connected across CON2) has UART interfaces with direct connections to the MCU or to the PC through max 232/USB serial adaptor. The user can store fingerprint data in the module and configure it in 1:1 or 1: $\mathrm{N}$ mode. For identification FP module can directly interface with $3 \mathrm{v} 3$ or $5 \mathrm{v}$ microcontroller.

The module supports USART communication protocol. Here, USART protocol is used for communicating with the microcontroller. Transmit pin of module is connected to the receive pin of microcontroller. Receive pin should be connected to the transmit pin of microcontroller. VIN is $5 \mathrm{~V}$ and GND is connected to ground.

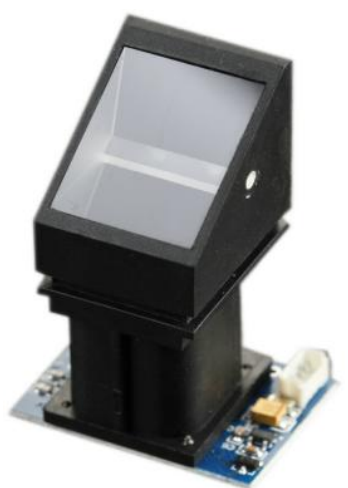

Figure 2: Fingerprint Sensor Module R305

\section{MICROCONTROLLER}

The project consists of 89C51 microcontroller of 8051 family microcontroller. It is 8 bit microcontroller and has 23 programmable input and output pins. It has $8 \mathrm{~KB}$ flash memory, 512 bytes of EEPROM, 1KB of SRAM. The microcontroller is a 40 pin IC. The IC and its pin configuration are shown Figure 3. 


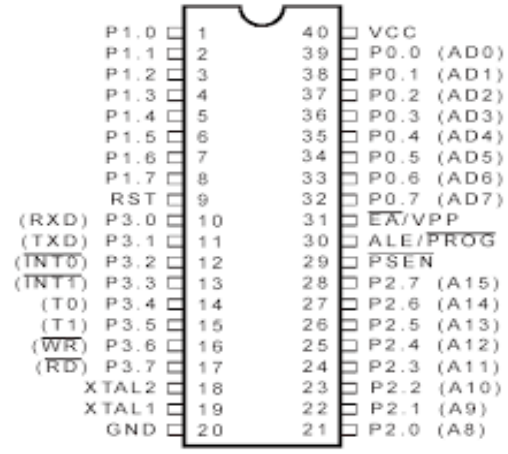

Figure 3: Pin Configuration of 89C51

In this system a $12 \mathrm{~V}$ adaptor is used to supply power. This input power is fed to a Voltage regulator - LM7805 which gives output of $5 \mathrm{~V}$. This is then supplied to the rest of the circuit. This board also includes a reset pin, a power LED, capacitors, resistor, and LCD and relay circuit. The circuit is shown in Figure 4. Embedded $\mathrm{C}$ is the language used to program the IC.

\section{LCD}

LCD (Liquid Crystal Display) screen is an electronic display module. A $16 \times 2$ LCD is very basic module. It can display 16 characters per line and there are 2 such lines. In a LCD display each character is displayed in $5 \times 7$ pixel matrix. The LCD is shown in Figure 5.

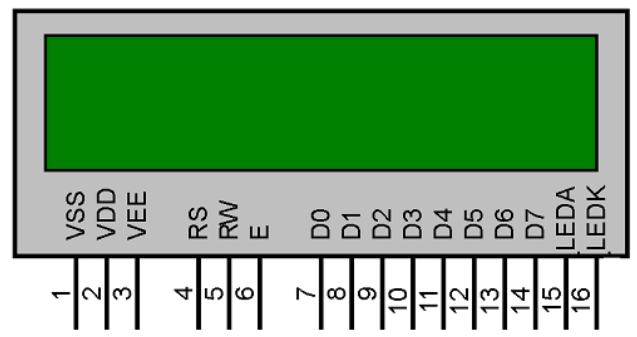

Figure 5: LCD display

This is interfaced to PORT A (pin 21-28) of the microcontroller. LCD in 8 bit mode is connected to microcontroller. D0-D7 pins are connected to PA08-PA15 pins of microcontroller. RW pin is connected to the GND.

\section{GSM MODEM}

Global System for Mobile Communication (GSM) is a wireless network system that uses a mobile operator and functions just like a mobile phone. The GSM modem has a SIM card slot, thus giving the modem a mobile number of its own and enabling it to activate communication. The user is able to send or receive a SMS as well as make/receive voice calls over the interface of the modem.
The GSM modem used in this project is SIM 900 module. It has a power and network LED which makes it convenient to debug. There is also wire antenna on the modem to provide better reception. The operating voltage range is $7-15 \mathrm{~V}$.

The ubiquity and low cost of implementation of the GSM standard makes it the ideal communication medium for a low budget security system. The GSM modem used in this device is shown in Figure 6.

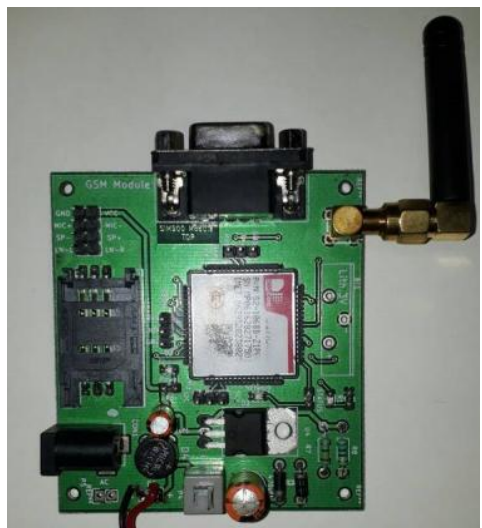

Figure 6: GSM Modem using SIM900 module

\section{RELAY CIRCUIT}

The type of relay used in this system is a Solid-State Relay (SSR). An SSR is basically an electronic switching device. Whenever an external voltage, over a certain threshold, is applied across its control terminals, a magnetic field is generated which attracts a movable lever, thus establishing a flow of current and switching power to a load circuitry. Thus, it functions as an electromechanical switch without any mechanical parts. But we have added a $600 \mathrm{rpm}$ DC motor to show the mechanical movement.

The relay circuit include resistors, two JQC-3FC/T73 relays working at $12 \mathrm{~V} \mathrm{DC}$ as shown in Figure 7. The maximum switching current for the relay is $7 \mathrm{~A}-10 \mathrm{~A}$.

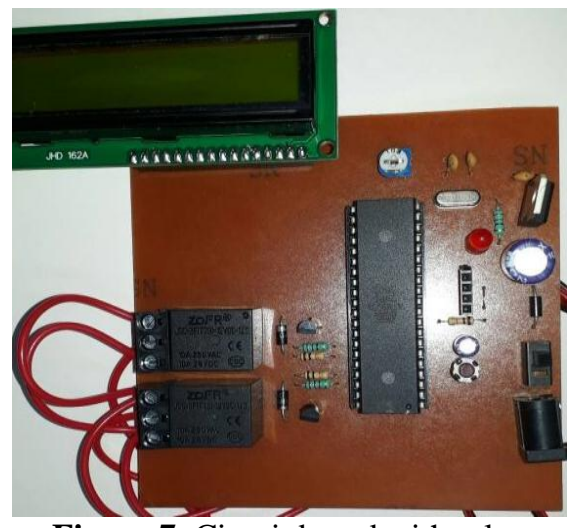

Figure 7. Circuit board with relay 
The relay is used to drive the DC motor. The two relays used rotate the motor in clockwise and anticlockwise direction respectively. The relay circuit is shown in Figure 8.

\section{PROBLEM FORMULATION}

During the course of the project, we came across several problems that were a hindrance to the appropriate functioning of the system. There were hardware as well as software issues. Out of many problems encountered, some of them are listed below.

\section{A. Complication with the GSM Modem:}

A $12 \mathrm{~V} / 1 \mathrm{~A}$ adaptor is needed to power up a GSM modem. After inserting a valid SIM card in the SIM card slot, the GSM catches network LED on GSM modem. It is connected to a tested microcontroller via a serial communication port.

However, in spite of having network the GSM modem was unable to send an SMS to the authorized person.

\section{B. Conflict with DC motor}

The relay used in the circuit is connected to the DC motor. The problem occurred was when system started working the motor used to rotate continuously. It was only stopped by cutting power supply from the system.

\section{PROPOSED PROBLEM SOUTION}

So as to ensure proper functioning of the system, the problems mentioned above were overcome as follows.

A. Solution for GSM Modem: The GSM Modem can be connected to the microcontroller. We connected the modem to the computer for debugging. The working of the GSM modem can be tested using the software terminal v1.9b. When we typing the command ' $\mathrm{AT}$ ' in command window, the terminal replies ' $\mathrm{OK}$ ' which indicates that the GSM modem is functioning properly. However, the terminal was not responding to this command. In order to overcome this problem, we used a $12 \mathrm{~V} / 2 \mathrm{~A}$ adaptor. This extra current, gave the modem boost necessary for proper functioning. After this, we had to switch back to the initial adaptor i.e. $12 \mathrm{~V} / 1 \mathrm{~A}$ adaptor and the GSM modem started functioning normally. The terminal has responded with ' $\mathrm{OK}$ ' and then showed all the AT commands being exchanged between the microcontroller and the modem.

B. Solution to the Soldering Issue: On testing the connectivity and the current flow in the soldered circuit with the help of a multimeter, we observed that a certain capacitor wasn't soldered properly. Opposite polarity had been soldered due to which the current reaching the IC exceeded the necessary level thus, spoiling the IC. Therefore, we had to resolder the capacitor and replace the IC for the proper functioning of the circuit.

\section{SYSTEM WORKING}

Fingerprint processing involves two steps finger enrolment and finger matching. Initially, to enroll the fingerprint, user must give his fingerprint twice to the module. Module checks these two images and generates a template image and stores it. In second step of finger matching, for 1 : $\mathrm{N}$ matching input is matched with the images in the library. It gives the matched image; a page id of the matched image is generated.

If the fingerprint is matched then buzzer will go on and signal goes to user and message on LCD will be "unauthorized person" and signal goes to the user. All these activities are informed to user with the help of message through GSM technology. GSM is second generation digital cellular mobile system used to send messages. GSM is also integrated to microcontroller to send message of activities.

\section{RESULTS AND ANALYSIS}

The Fingerprint Based Security System using GSM Module was successfully implemented.

\begin{tabular}{|l|l|}
\hline $\begin{array}{l}\text { Message on 16x2 LCD } \\
\text { Display }\end{array}$ & Interpretation \\
\hline System ready & $\begin{array}{l}\text { Fingerprint scanner } \\
\text { ready to scan finger }\end{array}$ \\
\hline Access Granted & $\begin{array}{l}\text { Fingerprint scanned is } \\
\text { verified and the motor } \\
\text { rotates in clockwise } \\
\text { direction. }\end{array}$ \\
\hline Access Denied & $\begin{array}{l}\text { Fingerprint scanned did } \\
\text { not match as on records } \\
\text { and a message is sent to } \\
\text { the registered mobile } \\
\text { number and the system } \\
\text { gets locked until } \\
\text { restarted }\end{array}$ \\
\hline Button & Function \\
\hline SEARCH & $\begin{array}{l}\text { Initiate the scanner } \\
\text { of a new user }\end{array}$ \\
\hline ADD & $\begin{array}{l}\text { Delete all registered } \\
\text { users }\end{array}$ \\
\hline EMPTY & Restart the entire system \\
\hline RESET & \\
\hline
\end{tabular}

\section{CONCLUSION}

In this project, a simple, user friendly, secure, low cost and universally acceptable solution for bank security has been introduced. This approach has enabled us to achieve the target of controlling 
the device remotely using a SMS-based system satisfying user needs and requirements. The system is cost effective as compared to the previously existing systems in market and is implemented with high reliability and security. The system is extendible and further additions can be done. Hence, we can conclude that the required goals and objectives have been achieved.

\section{REFERENCES}

[1]. Priya Darshini.V "Multilevel Security System for Automotives using RFID and Biometric Techniques in LabVIEW", International Journal of Advanced Research in Electrical, Electronics and Instrumentation EngineeringVol.

[2]. Issue 4, April 2013 2. Anil Jain, Arun Ross and Salil Prabhakar, "Fingerprint Matching Using Minutiae And Texture Features," Fingerprint Matching Using Minutiae And Texture Features", in Proc. of Int'l Conference on Image Processing (ICIP), pp.282-285, Thessaloniki, Greece, Oct 7 10,2001

[3]. www.nxp.com/lpc_2148 4. Rubella, J.A. "Fingerprint based license checking for auto-mobiles" Advanced Computing (ICoAC), 2012 Fourth International Conference 5 . www.synochip.com/en

[4]. Implementation ATM security by using fingerprint recognition and GSM by Pennam Krishna murthy \& Maddhusudhan reddy

[5]. Designing a biometric strategy(fingerprint) measure for enhancing ATM security in Indian E-Banking system-2011 by Sri Shimal Das smt. Jhunnu Deddarma

[6]. A method to improve the security level of ATM banking systems using AES algorithm, N. Selvaraj \& G. Sekar, international journal of computer applications (0975-8887) volume 3- no. 6 . June 2010.

[7]. Fingerprint recognition using minutia score matching by Ravi J K. B. Raja, Venugopal K. R Galton, F. Fingerprints. Mcmillan, 1982.

[8]. Mary Lourde $\mathrm{R}$ and Dushyant Khosla, "Fingerprint Identification in Biometric Security Systems" ,International Journal of Computer and Electrical Engineering, Vol. 2, No. 5, October, 2010.

[9]. Pramila D. Kamble, Dr.Bharti, W. Gawali, "Fingerprint Verification of ATM Security System by Using Biometric and Hybridization", International Journal of
Scientific and Research Publications, Volume 2, Issue 11, November 2012.

[10]. V.Ramya1, B. Palaniappan, V.Sumathi, "Gsm Based Embedded System For Remote Laboratory Safety Monitoring And Alerting". 